\title{
LOS ESTUDIOS DE HISTOLOGÍA ÓSEA EN ANTROPOLOGÍA BIOLÓGICA
}

\author{
Bárbara Desántolo ${ }^{1 *}$ y Valeria Bernal \\ ${ }^{\prime}$ Cátedra de Citología, Histología y Embriología “A”. Facultad de Ciencias Médicas. Universidad Nacional de La Plata. La Plata. Argentina \\ ${ }^{2}$ División Antropología. Facultad de Ciencias Naturales y Museo. Universidad Nacional de La Plata. La Plata. Argentina. Consejo Nacional \\ de Investigaciones Científicas y Técnicas (CONICET). Buenos Aires. Argentina
}

\section{PALABRAS CLAVE paleohistología, tejido óseo humano, microestructura}

\begin{abstract}
RESUMEN Los estudios sobre el tejido óseo de individuos actuales y prehistóricos brindan información única acerca de diferentes procesos ocurridos tanto durante la vida de los individuos -p. ej., patológicos, biomecánicos- como con posterioridad a su muerte -p. ej., tafonómicos-. En Argentina el análisis de la organización microestructural del tejido óseo en investigaciones bioantropológicas y bioarqueológicas presenta escasos antecedentes. En este número de la Revista
\end{abstract}

Argentina de Antropología Biológica se incluyen seis trabajos que reflejan la diversidad de los problemas y las técnicas histológicas empleadas en la actualidad. La selección de trabajos intenta proveer una mirada inclusiva de las áreas de aplicación del análisis de microestructuras óseas a fin de propiciar el fortalecimiento de líneas de investigación actualmente en desarrollo, así como la generación de nuevas líneas. Rev Arg Antrop Biol 18(2), 2016. doi:10.17139/raab.2016.0018.02.01

KEYWORDS palaeohistology, human bone tissue, microstructure

ABSTRACT Studies on bone tissue of both extant and prehistoric individuals provide unique information about different processes that occurred during the life of individuals -e.g. pathological, biomechanical- as well as after their death -e.g. taphonomic. In Argentina, the microstructural organization of bone tissue has been scarcely analyzed in bioarchaeological research. This issue of Revista Argentina de Antropología Biológica (Argentinean Journal of Biological Anthropology)

Los estudios sobre tejido óseo de individuos actuales y prehistóricos resultan de vital importancia en antropología biológica y forense, biología, biomedicina, arqueología y bioarqueología dado que en muchos casos éste constituye la única fuente de evidencia sobre procesos ocurridos tanto durante la vida de los individuos como con posterioridad a su muerte. Tradicionalmente, los estudios en antropología biológica han abordado el análisis esqueletal a una escala macro estructural -morfológica o anatómica- y más recientemente se han focalizado en la escala molecular. Comparativamente, una aproximación middle out en la escala del análisis histológico de rasgos microestructurales resulta aún poco explorada. Los datos obtenidos a esta escala permiten obtener información cualitativa y cuantitativa única -que no puede ser obtenida a partir de otras evidencias o resulta difícil de extraer a partir de restos fragmentados- para el abordaje de problemas paleopatológicos (Pfeiffer, 2000; DeBoer et al., 2013), de identificación de especies (Hillier y Bell, 2007), evaluación de la calidad ósea para estudios isotópicos contains six works that reflect the diversity of the issues addressed and the histological techniques employed in paleohistology. The selection of works provides an overview of the relevant contribution of bone micro-structures to various subjects with the aim of creating interest within the community of biological anthropologists and bioarchaeologists, and promoting the development of new lines of research. Rev Arg Antrop Biol 18(2), 2016. doi:10.17139/raab.2016.0018.02.01

y de ADN antiguo (ADNa), de variación morfológica en estudios ontogenéticos y evolutivos (Bromage, 1989), tafonómicos (Turner-Walker, 2008), paleodemográficos (Robling y Stout, 2008), y biomecánicos (Skedros et al., 2011). Empleados en conjunto, los datos histológicos y los provenientes de otras líneas de evidencia complementaria contribuyen al fortalecimiento de las inferencias realizadas.

En Argentina el estudio de la organización microestructural del tejido óseo para abordar problemas bioantropológicos y bioarqueológicos presenta escasos antecedentes que se restringen a contribuciones aisladas tendientes

\footnotetext{
Financiamiento: CONICET-PIP 0428; UNLP-PI 11/N742; UNLP-PI M189.

*Correspondencia a: Bárbara Desántolo. Cátedra de Citología, Histología y Embriología "A". Facultad de Ciencias Médicas. Universidad Nacional de La Plata. La Plata. Argentina. 122 y 60. 1900 La Plata. Argentina. E-mail: barbaradesantolo@hotmail.com
}

Recibido 6 Julio 2015; aceptado 11 Octubre 2015

doi:10.17139/raab.2016.0018.02.01 
a evaluar aspectos puntuales (Restelli et al., 1997, 1999; Gutierrez, 2001; Pan y Verri, 2002; Kozameh y Brunás, 2013). No obstante, estos estudios presentan gran potencial para generar nueva información y proveer perspectivas renovadas acerca de la biología esqueletal. La posibilidad de constituir un marco programático para la investigación en esta línea de trabajo -en el que se conecten preguntas específicas cuya respuesta requiera de la aplicación de métodos y técnicas de la histología- dependerá en gran medida de dos factores: la disponibilidad del equipamiento y el grado de entrenamiento que reciban los investigadores para el procesamiento y análisis de muestras histológicas. El primer factor no resulta una limitación dado que actualmente es posible acceder a diversos equipos a través del Sistema Nacional de Microscopía (Ministerio de Ciencia Tecnología e Innovación Productiva - Consejo Interinstitucional de Ciencia y Tecnología). El segundo factor requiere de mayores esfuerzos. En principio es necesaria la generación de vínculos de colaboración con especialistas en microscopía, análisis de imágenes, histología, bioquímica, entre otros. Asimismo, resulta imprescindible la incorporación de contenidos específicos tanto en los programas de las asignaturas de grado correspondientes a antropología biológica como en la formación de postgrado. En este sentido, durante 2014 se realizaron dos cursos dictados por la Dra. Martínez Mazza -Introducción al análisis paleohistológico de huesos: desarrollo, modo de vida y evolución en humanos y fauna asociada a yacimientos arqueológicos, Universidad Nacional del Centro- y por los Dres. Talevi, Cerda y García -Introducción a la Paleontología de vertebrados, Universidad Nacional de Río Negro-. La capacitación de investigadores en estas áreas permitirá que la información brindada por este tipo de análisis se incluya como información de base o estándar en investigaciones bioantropológicas y bioarqueológicas (Buikstra y Ubelaker, 1994).

Este número de la Revista Argentina de Antropología Biológica incluye trabajos de investigadores de Canadá, México, Argentina, Portugal y España con experiencia en el área de los estudios de histología ósea. El mismo intenta proveer una mirada inclusiva de la diversidad de problemáticas a las que el análisis de micro estructuras óseas puede contribuir y pretende generar interés en la comunidad de antropólogos biólogos y bioarqueólogos para propiciar la apertura de nuevas líneas de investigación. En particular, se discuten los avances de las técnicas de microscopía empleadas en estudios histológicos y sus aplicaciones a distintas problemáticas bioantropológicas y arqueológicas (Assis et al. y Barrientos et al.), se presentan estudios histomorfométricos basados en el análisis de cortes delgados para la estimación de edad de muerte en individuos adultos (Desántolo e Inda, y Suzuki y Tiesler) y estudios que emplean técnicas no invasivas para la descripción histológica de las áreas de formación y reabsorción en la superficie ósea (Brachetta Aporta et al., y Brachetta Aporta).

Assis y colaboradoras realizan una exhaustiva revisión histórica y recopilación bibliográfica de los estudios paleohistológicos sobre restos humanos de origen arqueológico y discuten las limitaciones actuales y desafíos futuros para el desarrollo de la paleohistología. En particular aquellas referidas al carácter invasivo y destructivo de algunas técnicas empleadas, el consumo de tiempo y dinero, y la falta de entrenamiento de los investigadores. Proponen incorporar técnicas menos invasivas -i.e. microscopio de luz polarizada y sincrotrón- y revisar los criterios de manejo de colecciones osteológicas entendiendo que la obtención de muestras para análisis histológicos constituye una transformación antes que una destrucción de las mismas.

El trabajo de Barrientos y colaboradoras efectúa una revisión crítica de los avances realizados en los estudios orientados a evaluar las modificaciones diagenéticas que generan una pérdida de la integridad histológica en material óseo de muestras arqueológicas. En particular, discuten las ventajas y desventajas de la aplicación de dos de los principales tipos de imágenes generadas por microscopía electrónica de barrido.

Desántolo e Inda realizan una revisión histórica de los métodos histomorfométricos basados en cortes delgados de huesos largos y resaltan la importancia de considerar la variación poblacional, la influencia de la composición sexual y etaria de las muestras empleadas como referencia, y la necesidad de estandarizar las observaciones de microestructuras óseas y de generar ecuaciones para la estimación de la edad específicas de cada población. Suzuki y Tiesler pre- 
sentan un análisis histomorfométrico de hueso no descalcificado para la estimación de la edad a la muerte a partir de costilla usando algoritmos desarrollados sobre una muestra documentada moderna de individuos mejicanos. Asimismo, proponen un nuevo método para cuantificar variables histomorfométricas en restos óseos que no presentan buen estado de conservación.

El trabajo de Brachetta Aporta y colaboradoras estudia los cambios ontogenéticos de la mandíbula mediante una perspectiva novedosa que combina la descripción histológica de las áreas de formación y reabsorción en la superficie del hueso con los aportes teóricos y metodológicos de la morfometría geométrica. Las autoras concluyen que los patrones descritos a escala histológica y anatómica exhiben gran similitud, aunque se detectan diferencias en cada escala, y por lo tanto, la información aportada por la histología y la morfometría seria complementaria. Finalmente, el trabajo de Brachetta Aporta representa el primer estudio sistemático del error de medición en el análisis paleohistológico centrado en la variación introducida por el investigador al observar réplicas de las superficies óseas al microscopio. Los resultados obtenidos remarcan la importancia de controlar esta fuente de variación y proveen un marco de referencia para evaluar la confiabilidad de las observaciones en futuros estudios paleohistológicos.

\section{AGRADECIMIENTOS}

A Ana Maria Inda, Ana Luísa Santos, Anne Keenleyside, Cayetana Martinez Maza, Gustavo Barrientos, Natalia Brachetta Aporta, Paula Galligani, Paula Gonzalez, Patricia Sarmiento, Sandra Assis, Shintaro Suzuki y Vera Tiesler por las contribuciones realizadas. Al equipo edi- torial de la Revista Argentina de Antropología Biológica.

\section{LITERATURA CITADA}

Bromage TG. 1989. Ontogeny of the early hominid face. J Hum Evol 18:751-773.

Buikstra J, Ubelaker D. 1994. Standards for data collection from human skeletal remains. Arkansas. Archaeological Survey Research Series 44.

De Boer HH, Van der Merwe AE, Maat GJR. 2013. The diagnostic value of microscopy in dry bone palaeopathology: a review. Int J Paleopath 3:113-121.

Gutierrez M. 2001. Bone diagenesis and taphonomic history of the Paso Otero 1 Bone Bed, Pampas of Argentina. J Archaeol Sci 28:1277-1290.

Hillier ML, Bell LS. 2007. Differentiating human bone from animal bone: a review of histological methods. J Forensic Sci 52:249-263.

Kozameh LF, Brunas OM. 2013. Enfermedad de Paget en un individuo prehispánico del Delta del Paraná, confirmado por examen histológico y datación radiocarbónica. Cuad Inst Nac Antropol series especiales 1(1):114-120.

Pan F, Verri S. 2002. Histomorfometría aplicada a restos óseos humanos arqueológicos: técnicas de desgaste vs meteorización. Cienc Morfol (La Plata) 6(9):3-16.

Pfeiffer S. 2000. Palaeohistology: health and disease. En: Katzenberg MA, Saunders S, editores. Biological anthropology of the human skeleton. New York: John Wiley \& Sons. p. 287-302.

Restelli M, Batista S, Bruno M, Salceda SA. 1999. Observaciones histológicas en hueso y diente provenientes de excavaciones arqueológicas. Cienc Morfol (La Plata) 3:25-37.

Restelli MA, Batista SL, Vasallo ML, Maliandi NE, Méndez MG, Salceda SA. 1997. Aportes de las técnicas micro y ultraestructurales sobre restos esqueletarios a la bioantropología. Actas II Jornadas Chivilcoyanas en Ciencias Sociales y Naturales de Chivilcoy. p. 123-128.

Robling AG, Stout SD. 2008. Histomorphometry of human cortical bone: applications of age estimation. En: Katzenberg MA, Saunders SR, editores. Biological anthropology of the human skeleton. New Jersey: John Wiley \& Sons. p. 149-182.

Skedros JG, Kiser CJ, Keenan KE, Thomas SC. 2011. Analysis of osteon morphotype scoring schemes for interpreting load history: evaluation in the chimpanzee femur. J Anat 218:480-499.

Turner-Walker, G. 2008. The chemical and microbial degradation of bones and teeth. En: Pinhasi R, Mays S, editores. Advances in human palaeopathology. Chichester: John Wiley \& Sons. p. 3-30. 